\title{
A Hybrid Technique for Shape Matching Based on chain code and DFS Tree
}

\author{
Kirti Jain ${ }^{1}$, Dr.Sarita Singh Bhadauria ${ }^{2}$, Dr.Gulab Singh ${ }^{3}$ \\ ${ }^{\text {I}}$ (Computer Science \&Engg Dept., LNCTS BHOPAL, INDIA) \\ ${ }^{2}$ (Electronics Dept.,M.I.T.S. GWALIOR, INDIA) \\ ${ }^{3}$ ( Computer Science \&Engg Dept , LNCTS BHOPAL,INDIA)
}

\begin{abstract}
Lower features content of image are playing a very important role for content based image retrieval. The lower features content of image are color, texture and shape. Shape finds outliers of object stored in database. The retrieval of image is used in many areas such as multimedia, medical science and internet technology. The semantic gap between query image and result of retrieval image. In this paper we propose a hybrid technique for shape matching based on chain code and DFS tree. Chain code is important and well method for boundary structured detection and DFS is graph searching technique. Here merge both method for detection of boundary pattern and matching of object. Used of DFS technique find the absolute detection of decide edge value of chain code.
\end{abstract}

Keywords: Shape Matching, Features Descriptors, chain Code, DFS

\section{Introduction}

Now the growth of technology and demand of multimedia data and medical diagnosis need a very high accurate result of content based image retrieval[1]. Content based image retrieval basically work on lower feature content of image and video. The lower content of image is playing an important role of image retrieval. Now a day's used for lower content of image is features descriptor such as MPEG-7 descriptor [4].MPEG-7 descriptor based on Fourier transform function for shape and boundary features detection. Many shape representation have been proposed for various purposes. These methods can generally be grouped into contourbased and region based. Contour-based methods, such as chain code, shape signature, polygonal approximation, autoregressive models, FD and CSS, exploit shape boundary information which is crucial to human perception in judging shape similarity[9]. Region based methods, such as geometric moments, Zernike moments, grid representation and area, exploit only shape interior information, therefore can be applied to more general shapes. The chain code shape detection have some disadvantage such as[5] (i) the chain of codes is too long (ii)Even slightly disturbed code would not reconstruct original image. Such problem faced retrieval for query image. These problem crate semantic gap between query image and retrieval image. For the reduction of chain code size used DFS searching technique. DFS reduced the size of code and produced better result in compassion of chain code. This paper is organized as follows: In Section 2, discuss features descriptors and chain code. Section 3 discusses DFS searching technique. In Section 4 discuss hybrid method of shape matching. In section 5 discuss experimental result. The final section makes the conclusion for this paper.

\section{Features Descriptors}

Features descriptor plays an important role in content based image retrieval and pattern Recognition[10]. Feature extraction can be defined as the act of mapping the image from image space to the feature space. Now days, finding good features that effectively represent an image is still a difficult task. In this literature, a wide variety of features are used for image retrieval from the database. Image content can differentiate between visual and semantic content. Features usually represent the visual content[9]. Visual content can be further divided into general or domain specific. Here discuss some shape based features descriptors .There are generally two types of shape representations, i.e. contour-based and region-based. Contour-based methods need extraction of boundary information which in some cases may not Available[3]. Region-based methods, however, do not necessarily rely on shape boundary information, but they do not reflect local features of a shape. Therefore, for generic purposes, both types of shape representations are necessary. Fourier descriptors are obtained by applying Fourier transform on shape boundary (usually represented by a shape signature), the Fourier transformed coefficients are called the Fourier descriptors of the shape. For good shape description, an appropriate shape signature is essential to obtaining Fourier descriptors.CSS (Curvature scale space)descriptors are essentially the descriptors of key local shape features. By dealing shape in scale space, not only the locations, but also the degree of convexities (or concavities) of shape boundaries are detected. Since curvature is a very important local measure of how fast a planar contour is turning, therefore, curvature scale space is exploited[7]. The CSS descriptors are obtained by first calculating the CSS contour 
map, the map is a multi-scale organization of the inflection points (or curvature zero-crossing points). Teague has proposed the use of orthogonal moments to recover the image from moments based on the theory of orthogonal polynomials, and has introduced Zernike moments, which allow independent moment invariants to be constructed to an arbitrarily high order. In grid shape representation, a shape is projected onto a grid of fixed size, $16 \times 16$ grid cells[4]. The grid cells are assigned the value of 1 if they are covered by the shape (or covered beyond a threshold) and 0 if they are outside the shape. In this paper discuss chain code method as a shape descriptor.

\section{Chain code}

A simple method to represent a contour is Freeman code (chain code)[5], a coding method of closed shape by approximation of the continuous contour with a sequence of numbers, each number corresponding to a segment direction[5]. Freeman's code is usually employed in a 4- neighborhood given in fig-1 (where 4 possible directions may be used) or in an 8-connectivity given in fig-2 (that gives an 8-directional chain code) [5]. This representation is compact and is invariant to the geometric transformation translation. However, it depends on rotation and scaling transformations. Moreover, it is difficult to manage Freeman's code with complex shapes.Chain code is a common approach for representing different rasterized shapes as line-drawings, planner curves, or contours. Chain code describes an object by a sequence of unit-size line segments with a given orientation. Chain code can be viewed as a connected sequence of straight -line segments with specified lengths and directions. Chain coding is used to define objects boundaries for the purpose of measurement and identification. A chain code follows the boundary of an object and indicates the change in direction according to a 4- or 8- direction pattern as shown here:

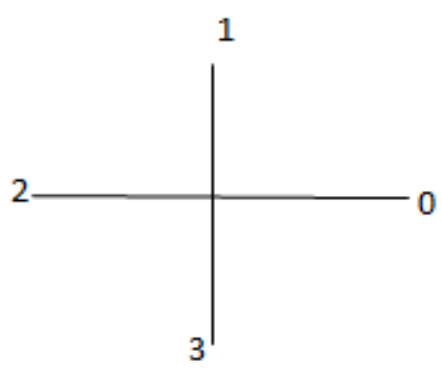

Fig-1[4-Connected]

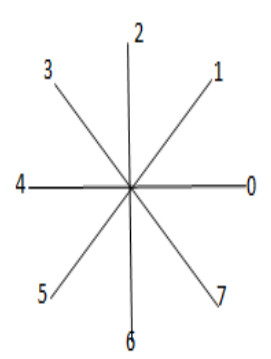

Fig-2 [8-Connected]

\section{DFS (Depth-First Search)}

As in previous section chain code method is used for boundary detection, but the problem with chain code is that it generate long chain code, if the shape is complex.However, it is also time consuming. In this paper Depth first search technique is used to reduce the size of long chain code. Depth-first search, as its name implies, to search "deeper" in the graph whenever possible. In depth-first search, edges are explored out of the most recently discovered vertex $u$ that still has unexplored edges leaving it[8]. When all of u's edges have been explored, the search "backtracks" to explore edges leaving the vertex from which u was discovered. This process continues until discovered all the vertices that are reachable from the original source vertex. If any undiscovered vertices remain, then one of them is selected as a new source and the search is repeated from that source. This entire process is repeated until all vertices are discovered. As in breadth-first search, whenever a vertex $\mathrm{u}$ is discovered during a scan of the adjacency list of an already discovered vertex $\mathrm{u}$, depth-first search records this event by setting u's predecessor field $\pi[\mathrm{u}]$ to $\mathrm{u}$. Unlike breadth-first search, whose predecessor subgraph forms a tree, the predecessor sub graph produced by a depth-first search may be composed of several trees, because the search may be repeated from multiple sources. The predecessor sub graph of a depth-first search is therefore defined slightly differently from that of a breadth-first search we let

$$
\mathrm{G},=(\mathrm{V}, \mathrm{E},) \text {, where }
$$

$$
\mathrm{E},=\{(\pi[\mathrm{v}] \mathrm{u},): \mathrm{u} \mathrm{E} \mathrm{V} \text { and } \pi[\mathrm{u}] \neq \mathrm{NIL}\} \text {. }
$$

The following pseudo code is the basic depth-first-search algorithm. The input graph $\mathrm{G}$ may be undirected or directed. The variable time is a global variable that we use for time stamping.

$\operatorname{DFS}(\mathrm{G})$

1 for each vertex u $\in \mathrm{V}[\mathrm{G}]$

2 do color $[\mathrm{u}] \leftarrow$ WHITE

$3 \pi[\mathrm{u}] \leftarrow \mathrm{NIL}$

4 time $\mathrm{t} \leftarrow 0$

5 for each vertex u $\epsilon$ V [G]

6 if $\operatorname{color}[u]==$ white 
7 then DFS-VISIT (U)

DFS-VISIT (U)

color $[\mathrm{u}] \leftarrow$ GRAY

$\mathrm{d}[\mathrm{u}] \leftarrow$ time $\leftarrow$ time +1

for each vertex $u \in \operatorname{Adj}[u]$

if $\operatorname{color}[\mathrm{v}]==$ WHITE

$\pi[\mathrm{v}] \leftarrow \mathrm{u}$

DFS - VISIT( v)

color $[\mathrm{u}] \leftarrow$ BLACK

$\mathrm{f}[\mathrm{u}] \leftarrow$ time $\leftarrow$ time +1

\section{Hybrid Technique}

In this paper as discussed in section II and III that chain code is using for boundary detection and DFS technique is used to overcome the problem of chain code method. Because here discuss chain code method with DFS technique to make result efficient and fast, therefore this method is called hybrid approach. Here chain code decides the direction of pixel data and depth-first search tree create a small loop of structure of sub graph. The generated sub graph produced the compactness of shape matching. The direction of chain code is too long. Now apply DFS and break long code into small loop sub graph, that sub graph produced a small segment of pattern. That segment of pattern produced better compactness of pattern matching. The applied chain code finds the length of boundary value and strore in vector as bit point. The vector point mapped the bit pattern black and white. The Order of relation of shape property is 1 to2. For a boundary counter value is $B_{i} \in C^{2}$ in image feature extraction process's $=1,2,3 \ldots . . . \mathrm{n}$ the level of traversing of spanning tree. Now the relation of pattern $\mathrm{P}(\mathrm{x}$, y) and mapped feature is $\mathrm{F}$.

$p(B i, F)=\sum_{(x \text { final }, y \text { final }) \in B i} \frac{[(x-x \text { final })+(y-y \text { final })] 1 / 2}{T(p)}$

Where (xfinal, yfinal) is the last point of chain length code. $T(p)$ is total visited level of path.

Here we present complete steps of our hybrid approach.

Step1: Create a binary image from the original image, possibly using some Thresholding and edge connection techniques.

Step2: Chose a random pixel of image and decide the source node of tree.

Step 3: Save the image in matrix of 0's for back ground and 1's for the object. While this process is in progress, we compute the area of the object by adding for each Iteration

Step 4: The visited node source to end of pixel is level wise and create array to stored

The visited link.

Step5: Compute chain code by scanning the image to find the starting pixel of the Object. From that pixel, we traverse the boundary and decide directions and save them as an array or list. This step is repeated until we reach the end pixel

If end pixel not equal start pixel then Shape is not closed

If end pixel =start pixel then

We have a closed shape

Proceed to step 4

Closed Opened

Step 6: If end of pixel found in level create a sub graph and generate small shape pattern.

Step7: Calculate the area of shape by summing up visited node sub graph.

Step8: Compute the compactness by the shape. Check these values to find out if shape is generated or not go to step 6.

Step 9: Count the corner based on direction change and save the elements for each Corner visited node. Elements are the 4-chain segments where direction changed. Check the angle in each corner. Depending on the number of corners and start and ending pixels. To decide the shape we have to find if the angles are 90

Step 10: Generate and connected sub graph produced many compacted small pattern.

Step 11: The transverse of DFS IS Stopped.

These are compact steps of our hybrid technique. From this technique we produced multiple patterns in single image. Through this approach the accuracy of compactness of pattern are increases.

\section{Experimental Result}

To implement the above technique, we first find the shape features for images. The number of pixels in each edge is referred to as one feature. These features are then stored in a matrix in row by row fashion i.e. each row contains the shape features corresponding to one image. In this paper evaluated performance of our algorithm using a general-purpose image database containing 500 JPEG images with size of $256^{\star} 256$ or 
$256^{*} 384$ pixels from COREL photo gallery. These images are divided into 5 categories, and there are 100 images in each semantic category. We test the performance of; the retrieval performance is measured by precision and recall, which are defined as

Precision $=\frac{\text { number of relevant images retrieved }}{\text { number of images retrieved }}$

Recall $==\frac{\text { number of relevant images retrieved }}{\text { number of relevant images in database }}$

Here we show the our retrieval result in figure this result is retrieved by our hybrid technique

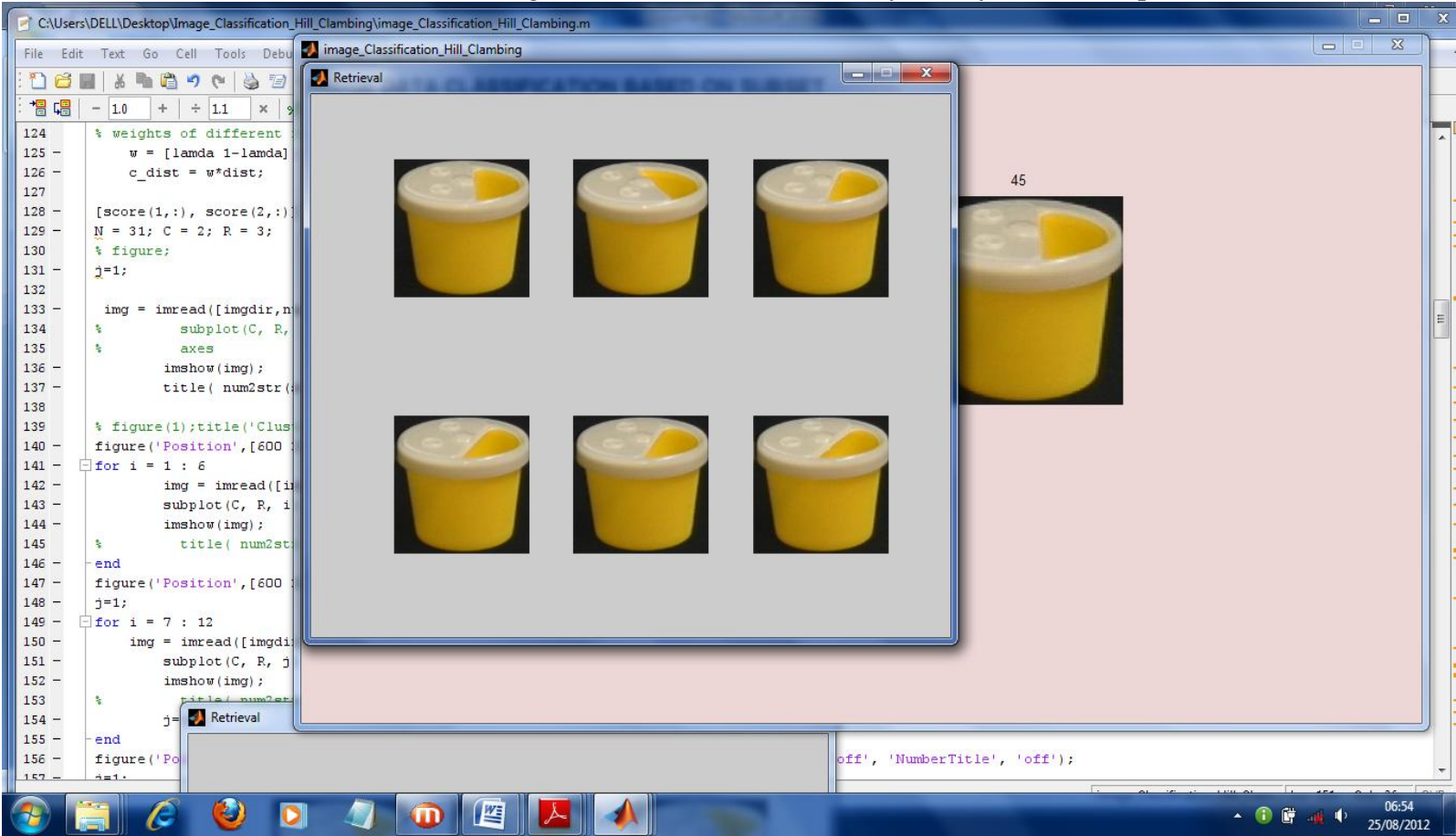

And the performance of retrieval is better in comparison of chain code technique.

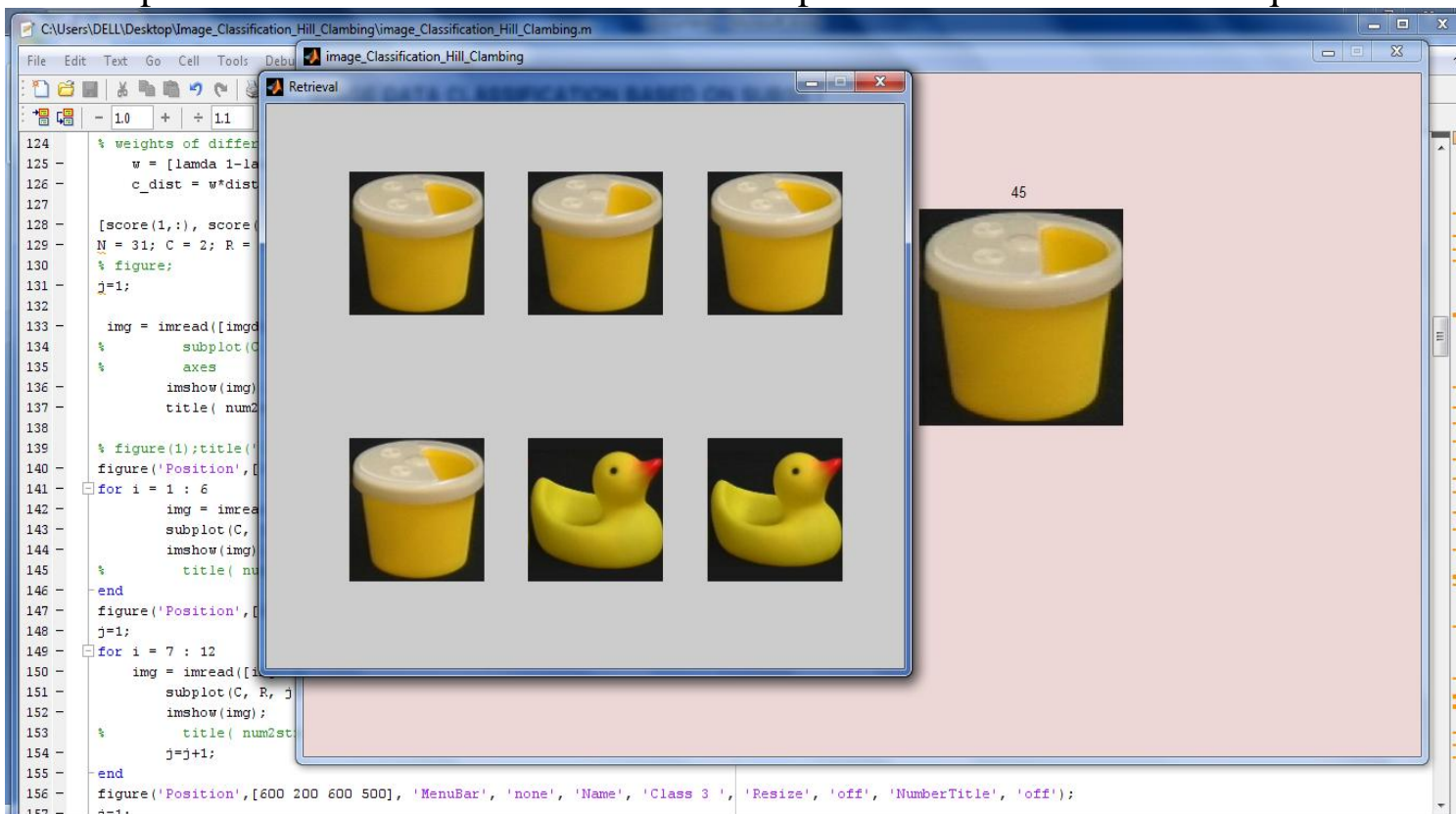

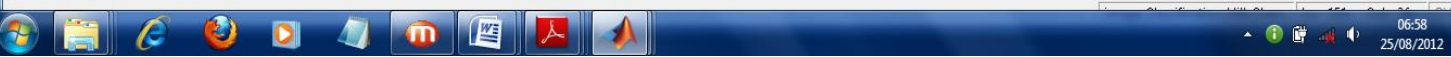

This figure shows that the retrieval result of chain code technique. 


\begin{tabular}{|c|c|c|c|}
\hline \multicolumn{2}{|c|}{ Data set } & \multirow{2}{*}{$\begin{array}{c}\text { Precision (\%) } \\
87.24\end{array}$} & \multirow{2}{*}{$\begin{array}{c}\text { Recall (\%) } \\
84.43\end{array}$} \\
\hline \multirow{2}{*}{ category 1} & $\begin{array}{l}\text { CHAIN } \\
\text { CODE }\end{array}$ & & \\
\hline & $\begin{array}{c}\text { HYBRIDE } \\
\text { DFS }\end{array}$ & 96.11 & 94.10 \\
\hline \multirow{2}{*}{ category 2} & $\begin{array}{l}\text { CHAIN } \\
\text { CODE }\end{array}$ & 84.32 & 83.23 \\
\hline & $\begin{array}{c}\text { HYBRIDE } \\
\text { DFS }\end{array}$ & 92.14 & 91.21 \\
\hline \multirow{2}{*}{ category 3} & $\begin{array}{l}\text { CHAIN } \\
\text { CODE }\end{array}$ & 86.14 & 85.11 \\
\hline & $\begin{array}{c}\text { HYBRIDE } \\
\text { DFS }\end{array}$ & 93.21 & 91.13 \\
\hline \multirow{2}{*}{ category 4} & $\begin{array}{l}\text { CHAIN } \\
\text { CODE }\end{array}$ & 88.21 & 87.66 \\
\hline & $\begin{array}{c}\text { HYBRIDE } \\
\text { DFS }\end{array}$ & 94.52 & 93.67 \\
\hline
\end{tabular}

Table 1 shows the empirical value of precision and recall

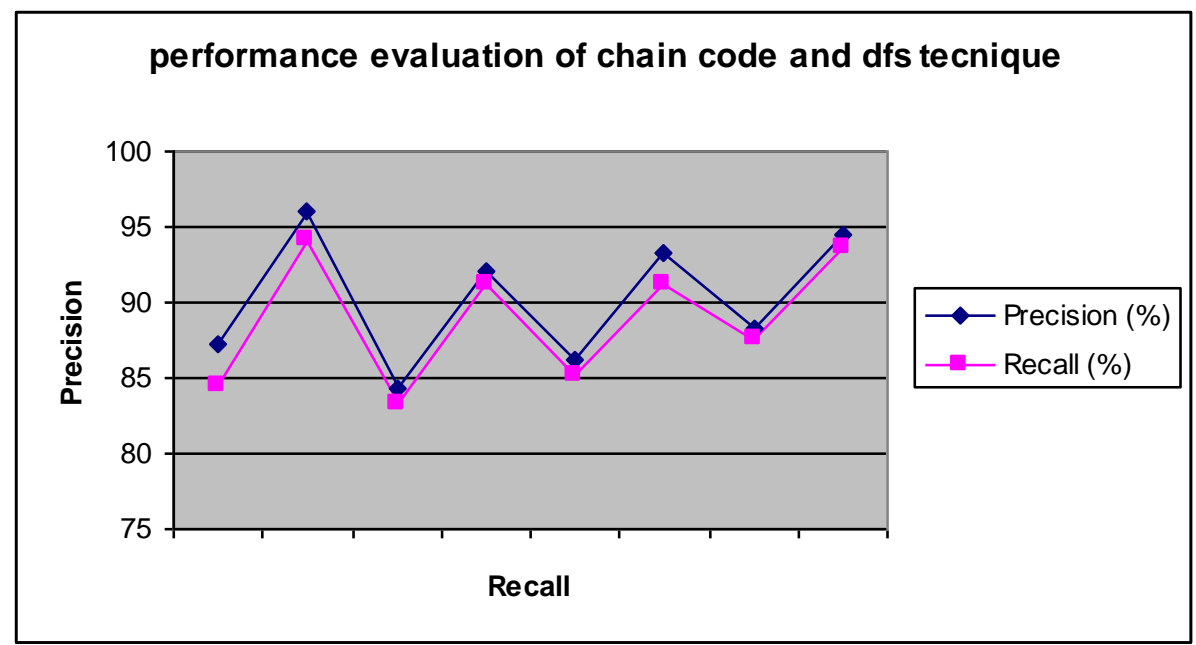

This graph shows that performance evaluation of both technique of image retrieval, blue line shows the dfs technique for the retrieval of image.

\section{Conclusion}

In this paper proposed a new technique for pattern detection using DFS technique combined with chain code method. These technique generate a sub graph of a small pattern and produced a better compactness and accuracy in compression of chain code method. Our experimental result shows the better performance in comparison of only used chain code method. Using hybrid approach the performance of precision and recall are increases in comparison of chain code method.

\section{References:}

[1] Eli Saber ,Yaowu Xub,, A. Murat Tekalp" Partial shape recognition by sub-matrix matching for partial matching guided image labeling" in Pattern Recognition 38 (2005) 1560 - 1573

[2] Dengsheng Zhang and Guojun Lu" A Comparative Study of Three Region Shape Descriptors" in Digital Image Computing Techniques and Applications, 21-22 January 2002

[3] Sanjoy K. Saha , Amit K. Das , Bhabatosh Chanda" Image retrieval based on indexing and relevance feedback" in Pattern Recognition Letters 28 (2007) 357-366

[4] Dengsheng Zhang, Guojun Lu" Evaluation of MPEG-7 shape descriptors against other shape descriptors " in Multimedia Systems c Springer-Verlag 2003

[5] Lili Ayu Wulandhari, Habibolah Haron," The Evolution and Trend of Chain Code Scheme" ICGST-GVIP, ISSN 1687-398X, Volume (8), Issue (III), October 2008.

[6] Naif Alajlan_, Mohamed S. Kamel, George Freeman" Multi-object image retrieval based on shape and topology" in Signal Processing: Image Communication 21 (2006)

[7] Dengsheng Zhang, Guojun Lu" Review of shape representation andd description techniques " in Pattern Recognition 37 (2004)

[8] Thomas H. Cormen Charles E. Leiserson Ronald L. Rivest” Introduction to Algorithms" in IEEE 2002 
[9] E. J. Cand 'es and D. L. Donoho, "Curvelets - a surprisingly effective nonadaptive representation for objects with edges," in Curve and SurfaceFitting, A. Cohen, C. Rabut, and L. L. Schumaker, Eds. Saint- Malo: Vanderbilt University Press, 2000.

[10] M. N. Do and M. Vetterli, "Contourlets," in Beyond Wavelets, G. V.Welland, Ed. New York: Academic Press, 2003.

[11] M. N. Do and M. Vetterli, "The Contourlet Transform: "An Efficient Directional Multiresolution Image Representation", IEEE Transactions on Image Processing, vol. 14, no. 12, December 2005, pp. $2091-2106$.

[12] D. D.-Y. Po and M. N. Do, "Directional multiscale modeling of images using the contourlet transform", IEEE Transactions on Image Processing, vol. 15, no. 6, pp. 1610-1620, June 2006.

[13] L. Zhang, P. Bao, "Edge detection by scale multiplication in wavelet domain", Pattern Recognition Letters", 2002, 23, pp, 17711784.

[14] Canny, J., 1986. “A computational approach to edge detection”. IEEE Trans. PAMI PAMI-8, pp. 679-698. 